\title{
Is rectal MRI beneficial for determining the location of rectal cancer with respect to the peritoneal reflection?
}

\author{
Eun Joo Jung ${ }^{1}$, Chun Geun Ryu¹, Gangmi Kim¹, Su Ran Kim¹, Sang Eun Nam¹, \\ Hee Sun Park², Young Jun Kim², Dae-Yong Hwang ${ }^{1}$
1 Department of Surgery, Colorectal Cancer Center, Konkuk University Medical Center, Konkuk University School of Medicine, Seoul, Republic of Korea
${ }^{2}$ Department of Radiology, Konkuk University Medical Center, Konkuk University School of Medicine, Seoul, Republic of Korea \\ Radiol Oncol 2012; 46(4): 296-301.
}

Received 24 January 2012

Accepted 12 May 2012

Correspondence to: Professor Dae-Yong Hwang, M.D., Department of Surgery, Colorectal Cancer Center, Konkuk University Medical Center; Konkuk University School of Medicine, 4-12 Hwayang-dong, Gwangjin-gu, Seoul, 143-729, Republic of Korea. Phone: +82-2-2030-5111; Fax : +82-2-2030-5112; E-mail : hwangcrc@kuh.ac.kr

Disclosure: No potential conflicts of interest were disclosed.

Background. An objective method for determining the location of the cancer with respect to peritoneal reflection would be helpful to decide the treatment modality for rectal cancer. This study was designed to evaluate the accuracy and usefulness of rectal MRI to determine spatial relations between the peritoneal reflection and rectal cancer and to compare these with operative findings.

Patients and methods. Patients that underwent a rectal cancer operation after a rectal MRI check between November 2008 and June 2010 were considered for the study. The patients that received preoperative concurrent chemoradiation or trans-anal local excision were excluded.

Results. Fifty-four patients constituted the study cohort. By comparing surgical and radiologic findings, the accuracy for predicting tumour location in relation to the peritoneal reflection by rectal MRI in all patients was $90.7 \%$. In terms of tumour location in relation to peritoneal reflection, the accuracy of rectal MRI was $93.5 \%$ in patients with a tumour located above the peritoneal reflection, $90.0 \%$ in patients with a tumour located on the peritoneal reflection, and $84.6 \%$ in patients with a tumour located below the peritoneal reflection $(p=0.061)$. When the cohort was subdivided by gender, body mass index (BMI), operative findings, or tumour size, no significant difference was observed among subgroups.

Conclusions. Rectal MRI could be a useful tool for evaluating the relation between rectal cancer and peritoneal reflection especially when tumour size is less than $8 \mathrm{~cm}$. Rectal MRI can provide information regarding the location of rectal cancer in relation to the peritoneal reflection for treatment planning purposes.

Key words: rectal cancer; peritoneal reflection; magnetic resonance imaging (MRI)

\section{Introduction}

Preoperative evaluations are of considerable importance for rectal cancer management, because treatment decision making is dependent on radiologic findings. Thus, neoadjuvant therapy could be determined based on preoperative clinical staging status, but it has not been determined which parts of rectal tumours should be included in such as staging system. Some authors have suggested that considerations of height from the anal verge might have beneficial on the radiotherapy of rectal tumors. ${ }^{1}$ However, measurements of distances from the anal verge are unclear because the methods devised to date, e.g., digital rectal examination or even rigid sigmoidoscopy, are rather subjective.

The peritoneal reflection is a landmark used for evaluating the rectum anteriorly, and divides the 
rectum into two parts, that is, the intraperitoneal and extraperitoneal regions, which are referred to considerations of the venous and lymphatic drainage systems of the rectum. ${ }^{2}$ In particular, extraperitoneal rectal tumours disseminate mainly through the systemic pelvic venous and lateral lymphatic drainage systems, whereas intraperitoneal rectal tumours disseminate mainly through the superior haemorrhoidal and inferior mesenteric venous and lymphatic drainage systems. ${ }^{2}$ Furthermore, Benzoni et al. concluded that tumour location in relation to the peritoneal reflection is a prognostic factor in rectal cancer. ${ }^{2}$ In this study, it was found that extraperitoneal rectal tumours are more aggressive than intraperitoneal tumours, even when treated by neoadjuvant chemoradiotherapy before surgery, which is common approach in the treatment of rectal cancer..$^{2-4}$ Thus, it appears that the peritoneal reflection might be useful for the adaptation of different treatment strategies in rectal cancer. Even though the definitions of intraperitoneal and extraperitoneal locations are ambiguous in relation to the mesorectum, it may be that the use of the peritoneal reflection as a discriminating structure in the pelvic cavity enables the differentiation of the locations of rectal tumours to the intraperitoneal and extraperitoneal regions.

Reported distances from the anal verge to the peritoneal reflection are highly variable. ${ }^{1,5}$ Accordingly, measurements based on this landmark cannot be used to precisely determine the location of the peritoneal reflection. We considered that if the peritoneal reflection could be clearly visualized and localized radiologically, that a more objective localization method could be devised than those based on distances from the anal verge.

Many reports have been issued on the role of magnetic resonance imaging (MRI) in rectal cancer in terms of determining circumferential margins or perirectal nodal statuses. ${ }^{6-8}$ However, few reports are available on the spatial relation between rectal cancer and the peritoneal reflection as determined by rectal MRI. Therefore, the aim of this study was to evaluate the accuracy and usefulness of rectal MRI for determining the relation between rectal cancer and the peritoneal reflection with respect to operative findings.

\section{Materials and methods}

Of the patients that underwent surgery for rectal cancer after a preoperative work-up (including rectal MRI) at the Colorectal Cancer Center, Konkuk

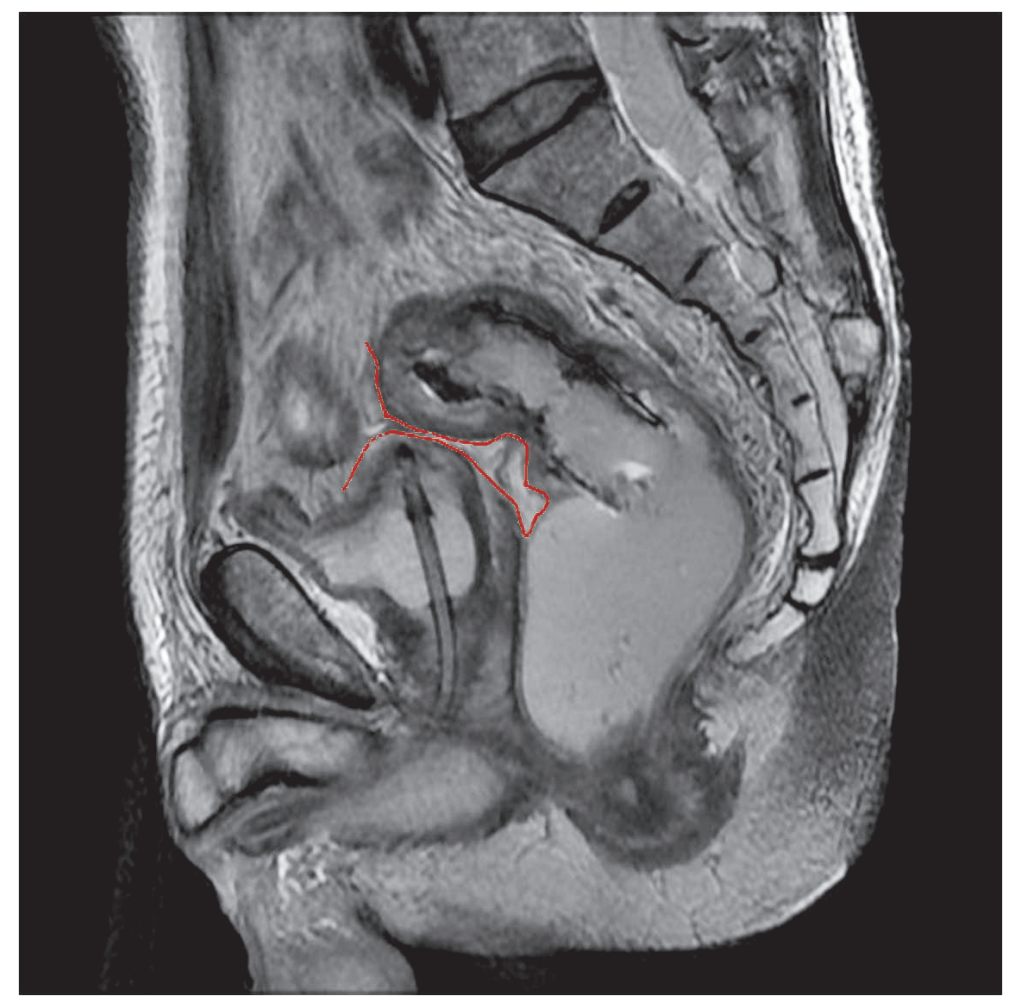

FIGURE 1. Sagittal view of the peritoneal reflection (red line) by rectal MRI.

University Medical Center between November 2008 and June 2010, 54 patients that did not receive preoperative concurrent chemoradiation or transanal local excision were included in the present study.

Rectal MRI images were reviewed by radiologists (H.S.P., and Y.J.K), on axial, sagittal, and coronal scans of T2-weighted images without clinical information. The peritoneal reflection appears as a low-signal-intensity linear structure that extends over the surface of the bladder and can be traced posteriorly to its point of attachment onto the rectum ${ }^{6}$ (Figure 1). Under the consensus decision, these two radiologists determined spatial relationships between rectal cancer and the peritoneal reflection, and allocated tumour locations to the following categories; a) a location completely proximal to the peritoneal reflection, $b$ ) a location mainly at the level of the peritoneal reflection, c) a location completely distal to the peritoneal reflection (Figure 2). In addition, tumour locations were categorized as; anterior, lateral, posterior, circumferential, anterolateral, or postero lateral.

Operative findings were recorded by a colorectal surgeon (DYH), who performed all surgeries. Intraoperative tumour levels were described by the surgeon as above, on, or below the peritoneal 


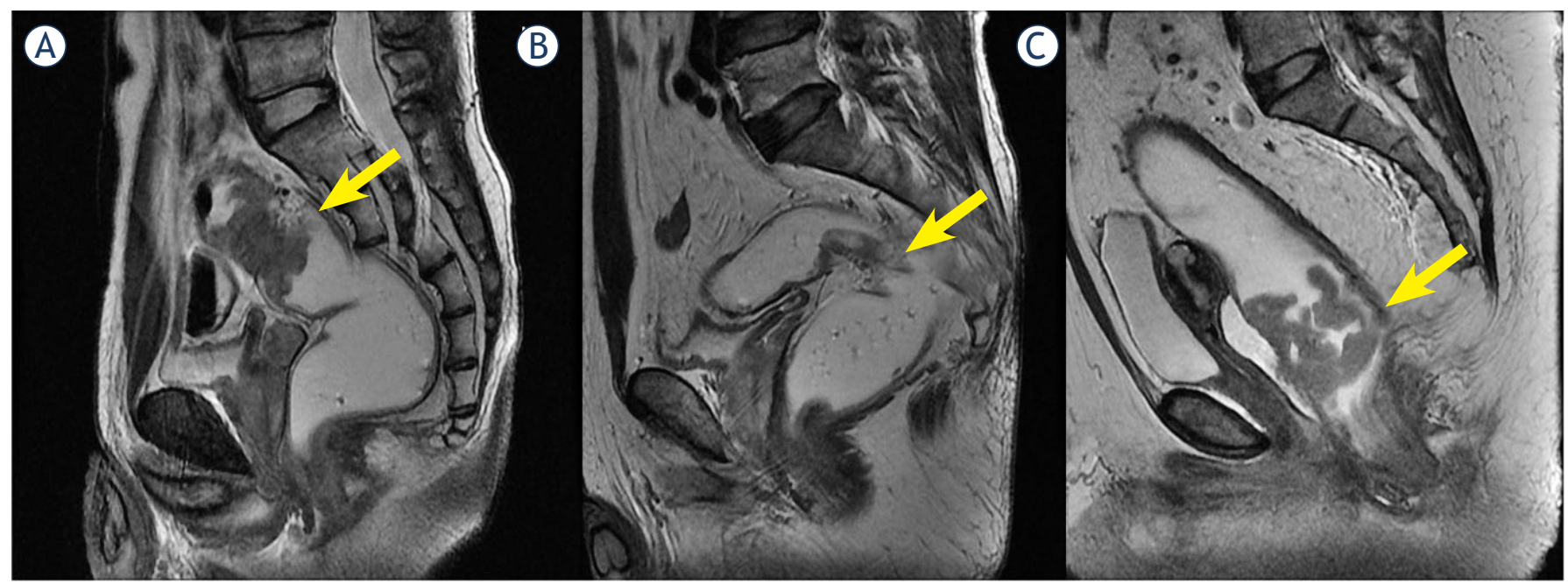

FIGURE 2. Tumour location with respect to the peritoneal reflection (PR). a. Tumour above the PR; b. tumour at the PR; c. tumour below the PR.

reflection, and as anterior, posterior, lateral, or circumferential. Tumour sizes were determined pathologically and tumours were staged according to the TNM staging system. Distances from the anal verge to lower tumour borders were determined by digital rectal examination and by sigmoidoscopy.

Body mass index (BMI) was calculated using weight $(\mathrm{kg})$ divided by height $(\mathrm{m})$ squared, and was divided into three groups; the low BMI group $\left(<20 \mathrm{~kg} / \mathrm{m}^{2}\right)$, the normal BMI group ( $\geq 20$ and $<25$ $\left.\mathrm{kg} / \mathrm{m}^{2}\right)$, and the high BMI group $\left(\geq 25 \mathrm{~kg} / \mathrm{m}^{2}\right)$.

Data analysis was performed using the 'Statistical Package for the Social Sciences (SPSS)' version 14.0 for Windows (SPSS, Inc. Chicago, IL). Pearson's chi-square test was used to compare locational accuracies between subgroups, and $p$-values of $<0.05$ were considered statistically significant.

\section{Results}

The 54 study subjects ( 32 males and 22 females) had a mean age of 62.2 years. All were diagnosed with adenocarcinoma and low anterior resection was performed in $49(90.7 \%)$ and abdominoperineal resection in $5(9.3 \%)$. TNM tumour stage was 0 in 2 patients (3.7\%), I in 11 patients $(20.4 \%)$, II in 17 patients (31.5\%), III in 22 patients $(40.7 \%)$, and IV in 2 patients (3.7\%). Mean tumour size was $4.8 \mathrm{~cm}$. Patients' characteristics are summarized in Table 1.

The accuracy of predicting tumour location relative to the peritoneal reflection by rectal MRI using surgical findings as the standard in all 54 patients was $90.7 \%$ (Table 2). No significant differences were found between gender, BMI, operative findings, and tumour size subgroups. The accuracy of predicting tumour location relative to the peritoneal reflection was $90.9 \%$ for males and $87.5 \%$ for females ( $p=0.092$ ); for BMI, accuracies were $88.0 \%$, $91.6 \%$, and $86.3 \%$ in the low, normal, and high BMI subgroups, respectively ( $p=0.528)$.

The accuracy of rectal MRI was $93.5 \%$ in patients with a tumour located above the peritoneal reflection, $90.0 \%$ in patients with a tumour located on the peritoneal reflection, and $84.6 \%$ in patients with tumour located below the peritoneal reflection ( $p=0.061$ ). When tumours were classified by 2 $\mathrm{cm}$ increments in size, accuracies were $88.9 \%$ for a tumour size of $0-1.9 \mathrm{~cm}, 91.7 \%$ for $2.0-3.9 \mathrm{~cm}, 93.3 \%$ for $4.0-5.9 \mathrm{~cm}, 100 \%$ for $6.0-7.9 \mathrm{~cm}$, and $57.1 \%$ for $8.0-10.0 \mathrm{~cm}(p=0.394)$, indicating that accuracy increased with tumour size until tumours exceeded $8 \mathrm{~cm}$.

In terms of predicting tumour direction (anterior, lateral, and posterior), the overall accuracy of rectal MRI was $44.4 \%$. No significant difference was observed between gender, BMI, operative findings, and tumour size subgroups. Overall, tumour directions were predicted less accurately than tumour locations (Table 3).

\section{Discussions}

When considering treatment options for rectal cancer, preoperative evaluations are important, because decisions regarding surgery and preoperative concurrent chemoradiotherapy are dependent on tumour location, mesorectal fascia involvement, 
TABLE 1. Demographics and clinical status of patients

\begin{tabular}{|c|c|c|c|}
\hline & & $N=54$ & $\%$ (range) \\
\hline Gender & $M: F$ & $32: 22$ & $59.3: 40.7$ \\
\hline Age(year) & & $62.2 \pm 10.8$ & $(41-84)$ \\
\hline Height $(\mathrm{cm})$ & & $161.0 \pm 9.6$ & $(137-175)$ \\
\hline Weight (kg) & & $63.4 \pm 12.3$ & $(41-107)$ \\
\hline $\mathrm{BMI}\left(\mathrm{kg} / \mathrm{m}^{2}\right)$ & & $23.1 \pm 3.1$ & $(17.4-42.3)$ \\
\hline Proportion of high preop. CEA & & 18 & 33.3 \\
\hline Proportion of high preop. CA19-9 & & 5 & 9.2 \\
\hline \multirow[t]{2}{*}{ OP Name } & LAR & 49 & 90.7 \\
\hline & APR & 5 & 9.3 \\
\hline \multirow[t]{4}{*}{ Cell type (differentiation) } & Well & 2 & 3.7 \\
\hline & Moderately & 48 & 88.9 \\
\hline & Poorly & 2 & 3.7 \\
\hline & Mucinous & 2 & 3.7 \\
\hline \multirow[t]{5}{*}{ TNM stage } & 0 & 2 & 3.7 \\
\hline & 1 & 11 & 20.4 \\
\hline & $\|$ & 17 & 31.5 \\
\hline & III & 22 & 40.7 \\
\hline & IV & 2 & 3.7 \\
\hline Tumour size $(\mathrm{cm})$ & & $4.8 \pm 2.5$ & $(0.9-10)$ \\
\hline No. of retrieved LNs & & $24.3 \pm 15.9$ & $(3-87)$ \\
\hline Distance from anal verge $(\mathrm{cm})$ & & $8.8 \pm 3.5$ & $(1-12)$ \\
\hline
\end{tabular}

Mean \pm standard deviation, $\mathrm{BMl}=\mathrm{body}$ mass index, preop. $=$ preoperative, $\mathrm{OP}=$ operation, $\mathrm{LN}=\mathrm{lymph}$ node, $\mathrm{LAR}=$ low anterior resection, $\mathrm{APR}=$ abdomino-perineal resection.

and nodal status. ${ }^{6,7,9-13}$ Rectal MRI is commonly performed preoperatively for evaluating the mesorectal fascia or adjacent organ involvement, and nodal staging. ${ }^{6-9}$ However, few reports have described the clinical usefulness of rectal MRI in terms of evaluating spatial relations between rectal tumours and the peritoneal reflection.

For descriptive purposes, the rectum is divided into three parts, that is, the upper, mid, and lower thirds. The upper third is covered by peritoneum anteriorly and laterally, whereas the middle third is covered only anteriorly, and the lower third is devoid of peritoneum ${ }^{9,10}$ In Japanese classification, the rectum is also divided into three parts, designated $\mathrm{Rs}, \mathrm{Ra}$, and $\mathrm{Rb} .{ }^{14}$ The border between $\mathrm{Ra}$ and $\mathrm{Rb}$ is defined to be at the level of the peritoneal reflection, which approximately corresponds to the level of the middle Houston valve. ${ }^{14}$ Thus, these classifications are based on the relation with respect to peritoneal reflection. However, it is not easy to determine the precise location of the peritoneal reflection preoperatively.
For this reason, most articles on rectal cancer define the upper rectum as 10 to $15 \mathrm{~cm}$ from the anal verge, the mid third as 5 to $10 \mathrm{~cm}$, and the lower third as $<5 \mathrm{~cm}$, although one author defined the upper third as 12 to $16 \mathrm{~cm}$ from the anal verge. ${ }^{15}$ However, this classification is vague and subjective, and reported distances from the anal verge based on these arbitrary divisions are not comparable.

Accordingly, we considered that the peritoneal reflection might be of use as a landmark to determine the location of the rectal subdivision for rectal cancer management. Some authors have evaluated the use of the peritoneal reflection as an anatomic landmark in rectal cancer patients. Gerdes et al. used trans-endorectal ultrasound (TRUS) to evaluate tumour locations with respect to the peritoneal reflection. ${ }^{16}$ In this study, the indicators of an intraperitoneal location were peristalsis beyond the rectal wall or intraperitoneal fluid collection. ${ }^{16}$ However, the study had two limitations, namely, that the peritoneal reflection could not be 
TABLE 2. Location of rectal cancer with respect to peritoneal reflection by radiologic and operative findings

\begin{tabular}{cccccc}
\hline No. of case & \multicolumn{5}{c}{ By surgeon } \\
\hline \multirow{5}{*}{ By radiologists } & Above PR & On PR & Below PR & Total \\
& Above PR & 29 & 1 & 0 & 30 \\
& On PR & 2 & 9 & 2 & 13 \\
& Below PR & 0 & 0 & 11 & 11 \\
& Total & 31 & 10 & & 54 \\
\hline
\end{tabular}

$\mathrm{PR}=$ peritoneal reflection

TABLE 3. Accuracies of predicting tumour directions and locations with respect to the peritoneal reflection

\begin{tabular}{|c|c|c|c|c|}
\hline & \multicolumn{2}{|c|}{$\begin{array}{l}\text { Prediction of } \\
\text { peritoneal reflection }\end{array}$} & \multicolumn{2}{|c|}{$\begin{array}{l}\text { Prediction of } \\
\text { tumour direction }\end{array}$} \\
\hline & Accuracy (\%) & $\mathbf{P}$ & Accuracy (\%) & $\mathbf{P}$ \\
\hline Gender & & 0.092 & & 0.561 \\
\hline Male $(n=32)$ & 90.9 & & 43.8 & \\
\hline Female $(n=22)$ & 87.5 & & 45.5 & \\
\hline BMI & & 0.528 & & 0.197 \\
\hline Low $\left(<20 \mathrm{~kg} / \mathrm{m}^{2}\right) \quad(\mathrm{n}=7)$ & 88.0 & & 48.3 & \\
\hline Normal $\left(20-25 \mathrm{~kg} / \mathrm{m}^{2}\right)(\mathrm{n}=36)$ & 91.6 & & 45.1 & \\
\hline High $\left(>25 \mathrm{~kg} / \mathrm{m}^{2}\right)(\mathrm{n}=11)$ & 86.3 & & 43.0 & \\
\hline Relationship with PR & & 0.061 & & 0.076 \\
\hline Above PR $(n=31)$ & 93.5 & & 38.7 & \\
\hline On PR $(n=10)$ & 90.0 & & 66.7 & \\
\hline Below PR $(n=13)$ & 84.6 & & 46.2 & \\
\hline Tumour size $(\mathrm{cm})$ & & 0.394 & & 0.462 \\
\hline $0 \sim 1.9(n=9)$ & 88.9 & & 66.7 & \\
\hline $2.0 \sim 3.9(n=12)$ & 91.7 & & 58.3 & \\
\hline $4.0 \sim 5.9(n=15)$ & 93.3 & & 46.7 & \\
\hline $6.0 \sim 7.9(n=11)$ & 100.0 & & 36.4 & \\
\hline $8.0 \sim 10.0(n=7)$ & 57.1 & & 28.6 & \\
\hline
\end{tabular}

$\mathrm{BMI}=$ body mass index, $\mathrm{PR}=$ peritoneal reflection

found in the absence of bowel peristalsis or fluid collection, and that trans-endorectal ultrasound is a practitioner-dependent subjective procedure. Others have also evaluated the usefulness of the peritoneal reflection as a landmark in rectal cancer by comparing intraoperative rigid proctoscopy and intraoperative findings. ${ }^{5,17}$ However, in these studies, data just suggested the location of peritoneal reflection or distance between the peritoneal reflection and anal verge, and in clinical practice, this data is not applicable for determining treatment plans in rectal cancer.
On rectal MR images, the peritoneal reflection appears as low-signal intensity linear structure at the junction between the rectum and the posterior aspect of the bladder in males or the vagina in females (Figure 1). In the present study, the accuracy of predicting the location of a rectal tumour with respect to the peritoneal reflection exceeded $88 \%$. Furthermore, clinical variables examined, such as gender or BMI, had no effect on this accuracy, but when the tumour size exceeded $8 \mathrm{~cm}$, accuracy fell to $57 \%$, presumably because large tumours disrupt the normal anatomy. 
In the present study, the accuracy of determining tumour direction was not high as $44.4 \%$, probably because natural rectal folds make the interpretation of direction difficult, although near the peritoneal reflection, the accuracy of tumour direction determination was rather high. However, as tumour size increased, it became more difficult to determine tumour direction. Nevertheless, the peritoneal reflection could be used to determine directions accurately for small size tumours less than $4 \mathrm{~cm}$.

In a Dutch study, it was suggested that upper third rectal cancer be treated like colon cancer ${ }^{18}$, and in a Dutch trial, no significant difference was found between a radiotherapy plus surgery group and a surgery only group in terms of local recurrence rates in upper rectal cancer. ${ }^{19}$ Lopez-Kostner et al. suggested that treatment outcomes for rectal cancer located 10 to $15 \mathrm{~cm}$ above the anal verge are similar to those of sigmoid colon cancer. ${ }^{20}$ At this time, no complete answer can be reached regarding whether upper third rectal cancer should be treated like colon cancer or rectal cancer. ${ }^{15}$ Nevertheless, recently, preoperative concurrent chemoradiotherapy has gained acceptance for the treatment of mid and lower rectal cancer. ${ }^{5}$

\section{Conclusions}

In conclusions, we believe that subdivision of the rectum by rectal MRI based on the location of the peritoneal reflection is more objective and anatomical than previously described methods, and that the more accurate information obtained regarding anatomic relations between rectal tumours and the peritoneal reflection aids treatment planning.

\section{References}

1. Memon S, Keating JP, Cooke HS, Dennett ER. A study into external rectal anatomy: improving patient selection for radiotherapy for rectal cancer. Dis Colon Rectum 2009; 52: 87-90.

2. Benzoni E, Terrosu G, Bresadola V, Cerato F, Cojutti A, Milan E, et al. Analysis of clinical outcomes and prognostic factors of neoadjuvant chemoradiotherapy combined with surgery: intraperitoneal versus extraperitoneal rectal cancer. Eur J Cancer Care 2006; 15: 286-92.

3. Lewander A, Gao J, Adell G, Zhang H, Sun XF. Expression of NF-kappa B p65 phosphorylated at serine-536 in rectal cancer with or without preoperative radiotherapy. Radiol Oncol 2011; 45: 279-84.

4. Mihaylova I, Parvanova V, Velikova C, Kurteva G, Ivanova D. Degree of tumor regression after preoperative chemo-radiotherapy in locally advanced rectal cancer-Preliminary results. Rep Pract Oncol Radiother 2011; 16: 237-42.

5. Yun HR, Chun HK, Lee WS, Cho YB, Yun SH, Lee WY. Intra-operative measurement of surgical lengths of the rectum and the peritoneal reflection in Korean. J Korean Med Sci 2008; 23: 999-1004.
6. Brown G, Kirkham A, Williams GT, Bourne M, Radcliffe AG, Sayman J, et al. High-resolution MRI of the anatomy important in total mesorectal excision of the rectum. AJR Am J Roentgenol 2004; 182: 431-9.

7. lafrate $F$, Laghi $A$, Paolantonio $P$, Rengo $M$, Mercantini $P$, Ferri $M$, et al. Preoperative staging of rectal cancer with MR imaging: Correlation with surgical and histopathologic findings. Raiographics 2006; 26: 701-14.

8. Tayler FGM, Swift RI, Blomqvist L, Brown G. A systemic approach to the interpretation of preoperative staging MRI for rectal ancer. AJR 2008; 191: 1827-1835.

9. Gordon PH, Santhat Nivatvongs. Principle and practice of surgery for the colon, rectum, and anus. 3rd edition. New York: Informa Health Care; 2006.

10. Townsend CM, Beauchamp RD, Evers BM, Mattox K. Textbook of surgery: The biologic basis of modern surgical practice. 17th edition. Phyladelphia: Elselvier Saunders; 2004.

11. Oblak I, Petric P, Andreluh F, Velenik V, Fras PA. Long term outcome after combined modality treatment for anal cancer. Radiol Oncol 2012; 46: $145-52$.

12. Ocvirk J. Advances in the treatment of metastatic colorectal carcinoma. Radiol Oncol 2009; 43: 1-8.

13. Conde $\mathrm{S}$, Borrego $\mathrm{M}$, Teixeira $\mathrm{T}$, Teixeira $\mathrm{R}$, Corbal $\mathrm{M}$, Sá $\mathrm{A}$, et al. Impact of neoadjuvant chemoradiation on pathologic response and survival of patients with locally advanced rectal cancer. Rep Pract Oncol Radiother 2010; 15: 51-9.

14. Japanese Society for Cancer of the Colon and Rectum. Japanese classification of colorectal carcinoma. Tokyo: Kanehara \& Co., LTD; 1997. p. 4-5.

15. Rosenberg R, Maak M, Schuster T, Becker K, Friess H, Gergler R. Does a recta cancer of the upper third behave more like a colon or a rectal cancer? Dis Colon Rectum 2010; 53: 761-70.

16. Gerdes B, Langer P, Kopp I, Bartsch D, Stinner B. Localization of the peritoneal reflection in the pelvis by endorectal ultrasound. Surg Endosc 1998; 12: 1401-4.

17. Najarian MM, Belzer GE, Cogbill TH, Mathiason MA. Determination of the peritoneal reflection using intraoperative proctoscopy. Dis Colon Rectum 2004; 47: 2080-5.

18. Peeters KCMJ, Marijnen CAM, Nagtegaal ID, Kranenbarg EK, Putter $\mathrm{H}$, Wiggers T et al. The TME trial after a median follow-up of 6 years: increased local control but no survival benefit in irradiated patients with resecTable rectal carcinoma. Ann Surg 2007; 246: 693-701.

19. Kapiteijn E, Marijnen CA, Nagtegaal ID, Putter H, Steup WH, Wiggers T, et al. Preoperative radiotherapy combined with total mesorectal excision for resecTable rectal cancer. N Engl J Med 2001; 345: 638-46.

20. Lopez-Kostner F, Lavery IC, Hool GR, Rybicki LA, Fazio VW. Total mesorectal excision is not necessary for cancers of the upper rectum. Surgery 1998; 124: $612-7$. 